Check for updates

Cite this: Nanoscale Adv., 2019, 1, 4772

\title{
Synthesis of mesoporous ceria using metal- and halogen-free ordered mesoporous carbon as a hard template $\uparrow$
}

\author{
Farzeen Sakina, ${ }^{a}$ Juan Manuel Muñoz-Ocaña, ${ }^{\mathrm{b}}$ Ainuona Bouziane, ${ }^{\mathrm{c}}$ Miguel Lopez- \\ Haro (iD) ${ }^{c}$ and Richard T. Baker (iD *a
}

\begin{abstract}
Ordered mesoporous cerias were synthesised by employing metal- and halogen-free ordered mesoporous carbons (OMCS) as the hard templates in a 'nanocasting' procedure. TEM, small angle (SA) and wide angle (WA) XRD, and $\mathrm{N}_{2}$ physisorption analyses were used to characterise the templates, intermediates and ceria products and electron tomography (STEM-HAADF) was used to explore the 3D morphology of the ceria nanostructures grown within the carbon templates. This allowed the relationships between the structures of the OMC templates and the products to be considered in detail as two parameters were varied. These were: the method of impregnation of the ceria precursor; and the temperature of calcination of the OMC template. Of the four impregnation methods tested, the solid-liquid method was found to be the most successful. This gave a high quality product with the highest yield of uniform mesopores, and crystalline nanorods of ceria arrayed in clear long-range order, as viewed by TEM and determined in SA and WAXRD. The specific surface area and pore volume exhibited by this sample were $111 \mathrm{~m}^{2} \mathrm{~g}^{-1}$ and $0.39 \mathrm{~cm}^{3} \mathrm{~g}^{-1}$, respectively. 3D electron tomography reconstructions revealed the presence of a network of ordered, nanoscale, rod-like structures interlinked in a complex fashion. The effect of calcination temperature of the template on uptake of the ceria precursor during impregnation was studied by calcining OMCs at temperatures from 350 to $800{ }^{\circ} \mathrm{C}$ and using these as hard templates for the nanocasting of ceria. Of these, the carbon template calcined at $400{ }^{\circ} \mathrm{C}$ gave the highest quality product.
\end{abstract}

rsc.li/nanoscale-advances

\section{Introduction}

Since the discovery of the first ordered mesoporous silica, MCM-41, the number of scientific studies on the synthesis, characterisation and application of ordered mesoporous metal oxides has increased strongly and continuously. ${ }^{1-6}$ Because of their very high specific surface area, large pore volume and adjustable pore size these materials are of great interest for various applications such as in catalysis and adsorption and separation processes. ${ }^{7}$ Mesoporous metal oxides may be synthesised using soft templating or hard templating - or "nanocasting" - methods. ${ }^{5}$ Soft templating typically involves the selfassembly of ordered arrays of surfactant micelles in a solution of a metal ion followed by calcination to remove the organics

${ }^{a}$ EaStChem School of Chemistry, University of St Andrews, St Andrews, Fife, KY16 9ST, UK. E-mail: rtb5@st-andrews.ac.uk; Tel: +44 (0)1334 463899

${ }^{b}$ Departamento de Estadística e Investigación Operativa, Facultad de Ciencias, Universidad de Cádiz, Campus Rio San Pedro, Puerto Real, Cádiz, 11510, Spain

'Departamento de Ciencias de los Materiales e Ingeniería Metalúrgica y Química Inorgánica, Facultad de Ciencias, Universidad de Cádiz, Campus Rio San Pedro, Puerto Real, Cádiz, 11510, Spain

$\dagger$ Electronic supplementary information (ESI) available. See DOI: $10.1039 / \mathrm{c} 9 \mathrm{na} 00482 \mathrm{c}$ and leave an ordered mesoporous metal oxide. ${ }^{5,8}$ Although the soft templating method has been very successful in the preparation of highly ordered mesoporous silicas (SBA-15, KIT-6, FDU-12 and SBA-16 etc.) and carbon materials (FDU-14, FDU15 and FDU-16 etc.), the synthesis of mesoporous forms of many metal oxide compositions is often not possible because of their thermal instability during the removal of the template., Furthermore, mesoporous metal oxides synthesised using the soft template method may suffer from amorphous (rather than crystalline) material in the pore walls and poor mesostructures, resulting in thermal and mechanical instability, which is undesirable in most applications, such as in catalysis. ${ }^{7,10}$ The soft-templating method can also give rise to materials containing macroscopic, or grain, heterogeneities. ${ }^{\mathbf{1 1}}$ For example, SBA-type materials such as SBA-15 show worm-like phases as well as the desired phase containing hexagonally-ordered channels. Materials made using nanocasting are reported not to contain such heterogeneities even when they are not highly ordered. ${ }^{11}$ Lastly, it is often difficult to remove the silica material entirely from the product, especially if it is also an oxide, such as zirconia. ${ }^{12}$ The use of carbon-based templates, which can be completely removed by combustion, is therefore an attractive alternative strategy. 
In hard templating, an already-prepared mesoporous material, such as mesoporous silica or carbon, is used as a template to form other useful mesoporous materials. The pores of the hard template are impregnated with a precursor of the desired final material using one of a number of impregnation methods including incipient wetness (IW), immersion of the template in bulk solution and the so-called solid-liquid (SL) method. After impregnation, the precursor is thermally decomposed to form the respective metal oxide. ${ }^{9}$ The hard template is then removed by dissolution or combustion, to yield the desired mesoporous metal oxide, whose structure is an inverse replica of that of the template. ${ }^{9}$ Highly ordered mesoporous metal oxides with crystalline wall material and having thermal stability can be synthesised using this method. ${ }^{\mathbf{9 , 1 0 , 1 3 , 1 4}}$

Cerium oxide has been widely investigated because of its catalytic activity and oxygen storage and release ability. ${ }^{15}$ It is used in three-way automobile exhaust catalysts, in the catalytic combustion of various hydrocarbons and for removing organics from polluted water ${ }^{7,10,15-19}$ It is clear that the catalytic activity of ceria-based materials might be enhanced by preparing it in mesoporous form with a uniform pore structure. ${ }^{15}$

Ryoo and Laha first reported the synthesis of highly ordered mesoporous $\mathrm{CeO}_{2}$ using SBA 15 and KIT-6 as the hard templates, $\mathrm{CeCl}_{3} \cdot 7 \mathrm{H}_{2} \mathrm{O}$ as the ceria precursor and the IW method of impregnation. The mesoporous $\mathrm{CeO}_{2}$ product obtained had uniform pore sizes with nanocrystalline pore walls and exhibited high thermal stability, even at $700{ }^{\circ} \mathbf{C} .^{5,15}$ Shen et al. made mesoporous $\mathrm{CeO}_{2}$ using KIT-6 as the hard template and $\mathrm{Ce}\left(\mathrm{NO}_{3}\right)_{3} \cdot 6 \mathrm{H}_{2} \mathrm{O}$ as the ceria precursor and used it to support $\mathrm{CuO}$ for the catalytic oxidation of $\mathrm{CO} .{ }^{10} \mathrm{Ji}$ et al. used MCM-48 with Ia3d symmetry as the hard template to prepare mesoporous ceria which showed a blue shift in UV-visible absorbance and was found to have a high concentration of surface oxygen vacancies. This material showed better photocatalytic activity for the decomposition of acid orange 7 (a nonbiodegradable azo dye and target contaminant) than corresponding nonporous analogues and standard $\mathrm{TiO}_{2}$ reference materials. $^{20}$ Wang et al. used two different kinds of silica templates, SBA-15 rods and spheres, to synthesised mesoporous $\mathrm{CeO}_{2}$ with different morphologies, such as micrometre-sized rod, hollow sphere, solid sphere and saucer-like structures, by carefully controlling the loading of the precursor. According to the authors, these differences in the textural properties of the mesoporous products when prepared using the same template could be attributed to the properties of the metal precursors and the interaction between the metal oxide and silica. ${ }^{7}$ Yue et al. prepared single crystal mesoporous ceria by using SBA-15, KIT-6, ${ }^{21}$ FDU-12 and SBA-16 ${ }^{\mathbf{9 , 1 4}}$ using the SL method for infiltration of the precursors. Tiemann et al. synthesised mesoporous ceria with crystalline walls using the mesoporous carbon, CMK-3, as the hard template and the IW method for impregnation of the ceria precursor, which was $\mathrm{Ce}\left(\mathrm{NO}_{3}\right)_{3} \cdot 6 \mathrm{H}_{2} \mathrm{O}$. The resulting product was used as a catalyst for decomposition of methanol., ${ }^{522}$ Wencui and co-workers prepared mesoporous ceria using CMK-3 as the hard template and used it as a support for the preparation of gold catalysts by the colloid deposition method. The authors found enhanced catalytic activity of $\mathrm{Au}$ /
$\mathrm{CeO}_{2}$ catalyst for $\mathrm{CO}$ oxidation. ${ }^{23}$ Garcia and co-workers used mesoporous ceria templated from KIT-6 as a catalyst for total oxidation of naphthalene. ${ }^{24}$ Recently, Solsona and coworkers tested the catalytic activity of mesoporous $\mathrm{CeO}_{2}$ and $\mathrm{Cu}$-loaded $\mathrm{CeO}_{2}$ made using several methods, including nanocasting from CMK-3, for total oxidation of propane..$^{25}$

Herein we report synthesis of ordered mesoporous $\mathrm{CeO}_{2}$ using as the hard template a metal- and halogen-free ordered mesoporous carbon (OMC) with a 2-D hexagonal arrangement of pores prepared as part of a previous study. ${ }^{26}$ The avoidance of metal and halogen species - often used in the conventional preparation of OMCs - is an important benefit since otherwise these can persist in the final product and act as catalyst poisons. We believe that this method could be used generally to prepare products of a wide range of compositions. An important requirement for nanocasting is the successful filling of the pores of the template with the precursor. To find the best conditions for this, two parameters were varied in this research. First, the effect of impregnation method was studied by testing four different methods: incipient wetness impregnation (IW), concentrated solution (CS), dilute solution (DS) and SL methods. Second, the chemical nature of the carbon template itself - and importantly of the pore walls - was modified by calcining the templates at different temperatures, $T_{\text {calc }}$. The ceria precursor used in all experiments was $\mathrm{Ce}\left(\mathrm{NO}_{3}\right)_{3} \cdot 6 \mathrm{H}_{2} \mathrm{O}$.

\section{Experimental}

\subsection{Synthesis of the standard mesoporous carbon template}

The 2D hexagonal OMC hard templates were prepared using a two phase method with the reagents in the molar ratios: resorcinol (R) : formaldehyde (F) : Pluronic F127 (a block copolymer) : $\mathrm{NH}_{4^{-}}$ $\mathrm{OH}:$ oxalic acid $=1: 1.39: 0.00635: 0.01: 0.125$. In a typical procedure, a precursor solution of resorcinol and formaldehyde was made by mixing $2.20 \mathrm{~g}$ of resorcinol with $2.26 \mathrm{~g}$ of $37 \mathrm{wt} \%$ formaldehyde solution for $1 \mathrm{~h}$ at $35^{\circ} \mathrm{C} .2 \mathrm{ml}$ of $0.1 \mathrm{M} \mathrm{NH}_{4} \mathrm{OH}$ was added and the solution was stirred for a further $1 \mathrm{~h}$ to obtain the $\mathrm{RF}$ resol. This was cooled to $18{ }^{\circ} \mathrm{C}$ for $10 \mathrm{~min}$ and was mixed with a solution containing $1.60 \mathrm{~g}$ of F127, $8 \mathrm{~g}$ of water and $10 \mathrm{~g}$ of ethanol for $20 \mathrm{~min}$ and $0.225 \mathrm{~g}$ of oxalic acid was added with continuous stirring. After 5-10 min, the clear solution became cloudy indicating phase separation. After constant stirring for a further $1 \mathrm{~h}$, the mixture was left standing overnight to obtain the polymer gel phase. This polymer gel was dried for at least $12 \mathrm{~h}$ at room temperature and cured at $80{ }^{\circ} \mathrm{C}$ for $24 \mathrm{~h}$. The resultant material was finally calcined at $400{ }^{\circ} \mathrm{C}$ for $3 \mathrm{~h}$ with a heating rate of $1{ }^{\circ} \mathrm{C} \mathrm{min}{ }^{-1}$ under $\mathrm{N}_{2}$ atmosphere $\left(100 \mathrm{~cm}^{3} \mathrm{~min}^{-1}\right)$ to obtain the standard OMC template. Full preparation and characterisation details of this and related materials are available elsewhere. ${ }^{26}$

\subsection{Synthesis of mesoporous ceria}

\subsubsection{Impregnation methods}

2.2.1.1 Solid-liquid method (SL). $2 \mathrm{~g}$ of the standard carbon template (calcined at $400{ }^{\circ} \mathrm{C}$ ) was ground with $\mathrm{Ce}\left(\mathrm{NO}_{3}\right)_{3} \cdot 6 \mathrm{H}_{2} \mathrm{O}$ powder in a pestle and mortar for about $10 \mathrm{~min}$. The volume of $\mathrm{Ce}\left(\mathrm{NO}_{3}\right)_{3} \cdot 6 \mathrm{H}_{2} \mathrm{O}$ used was equal to the pore volume of the 
carbon template used. In the literature, this is called the solidliquid (SL) method because the nitrate melts in the subsequent heating step (Section 2.1.2) and so is able to enter the mesopores of the template. ${ }^{21}$

2.2.1.2 Incipient wetness method (IW). A saturated solution in ethanol containing $\mathrm{Ce}\left(\mathrm{NO}_{3}\right)_{3} \cdot 6 \mathrm{H}_{2} \mathrm{O}$ which, when crystallised, would have a volume equal to the pore volume of ordered mesoporous carbon used, was added stepwise to $2 \mathrm{~g}$ of the standard OMC template. In each step, precursor solution was added dropwise and mixed thoroughly. The whole process took about $1 \mathrm{~h}$ after which the sample was left to dry overnight at room temperature.

2.2.1.3 Concentrated solution method (CS). In this method, $2 \mathrm{~g}$ of the standard carbon template was mixed with $10 \mathrm{ml}$ of $2 \mathrm{M}$ $\mathrm{Ce}\left(\mathrm{NO}_{3}\right)_{3} \cdot 6 \mathrm{H}_{2} \mathrm{O}$ in ethanol and stirred for $24 \mathrm{~h}$ in a centrifuge tube. The excess $\mathrm{Ce}\left(\mathrm{NO}_{3}\right)_{3} \cdot 6 \mathrm{H}_{2} \mathrm{O}$ solution was then removed by centrifugation and the product dried at room temperature overnight.

2.2.1.4 Dilute solution method (DS). An amount of Ce $\left(\mathrm{NO}_{3}\right)_{3} \cdot 6 \mathrm{H}_{2} \mathrm{O}$ equal in volume - when crystallised - to the pore volume of the carbon template was dissolved in $20 \mathrm{ml}$ of ethanol in a small beaker. $2 \mathrm{~g}$ of the standard carbon template was added and the mixture was stirred at $60{ }^{\circ} \mathrm{C}$ on a hotplate until the ethanol had evaporated and the sample was left to dry at room temperature overnight.

2.2.2 Calcination. After each impregnation method, all samples were heated to $200{ }^{\circ} \mathrm{C}$ in air at a rate of $1{ }^{\circ} \mathrm{C} \mathrm{min}{ }^{-1}$, kept at this temperature for $2 \mathrm{~h}$ then allowed to cool. A portion of each of these samples (denoted Intermediate Products, "IP") were kept separate for characterisation while the remainder was calcined first at $320^{\circ} \mathrm{C}$ for $3 \mathrm{~h}$ (heating rate of $1{ }^{\circ} \mathrm{C} \mathrm{min}{ }^{-1}$ ) and then at $530{ }^{\circ} \mathrm{C}$ for $1 \mathrm{~h}$ (ramp rate of $1{ }^{\circ} \mathrm{C} \mathrm{min}{ }^{-1}$ ) to give the final ceria products (denoted as “Ceria”s). The IPs and ceria products are named according to the impregnation method used in their preparation, SL-IP, SL-Ceria etc.

2.2.3 Variation of the calcination temperature of the OMC template. In addition to the ceria materials made using the standard template, the SL method was employed to prepare mesoporous ceria products using carbon templates prepared as in Section 2.1 but calcined at a series of temperatures, $T_{\text {calc }}=$ $350{ }^{\circ} \mathrm{C}, 400{ }^{\circ} \mathrm{C}, 500{ }^{\circ} \mathrm{C}, 600{ }^{\circ} \mathrm{C}$ and $800{ }^{\circ} \mathrm{C}$. All other preparation conditions were unchanged.

\subsection{Characterisation}

Small angle $\left(2 \theta=0.5\right.$ to $\left.5^{\circ}, \mathrm{SA}\right)$ and wide angle $\left(2 \theta=10\right.$ to $90^{\circ}$, WA) powder X-ray diffraction (XRD) patterns were recorded using a Panalytical Empyrean diffractometer in reflectance geometry using $\mathrm{Cu} \mathrm{K} \alpha$ radiation for $1 \mathrm{~h}$ with a step size of $0.017^{\circ}$. Transmission Electron Microscopy (TEM) was performed on a JEOL JEM 2011 instrument operating with a $\mathrm{LaB}_{6}$ filament at an accelerating voltage of $200 \mathrm{kV}$. TEM samples were prepared by suspending ground mesoporous samples in acetone by ultrasonication for $1 \mathrm{~min}$, sweeping a 300-mesh holey carbon TEM grid through the suspension and allowing this to dry under a halogen lamp overnight. Electron Tomography (ET) experiments were performed in an aberration- corrected FEI Titan $^{3}$ Themis 60-300 microscope working in the scanning transmission mode (STEM) at $200 \mathrm{kV}$. Experimental High Angle Annular Dark Field (HAADF) image series were acquired in the $-70^{\circ}$ to $+70^{\circ}$ tilt angle range, with $5^{\circ}$ steps. A convergence angle of $9 \mathrm{mrad}$ was selected in order to improve the depth of focus and a camera length of $115 \mathrm{~mm}$ was used. The ET series acquisition was done automatically using the FEI Explore3D v.4.1.2 software. To denoise the tilt series, homemade scripts coded in MATLAB were used based on the WAVELAB850 toolbox (http://statweb.stanford.edu/ wavelab/ Wavelab_850/index_wavelab850.html) and the invansc package downloaded from http://www.cs.tut.fi/ foi/invansc/. The whole set of HAADF- STEM denoised images were aligned by combining a cross-correlation method, using FEI Inspect3D v 3.0 and the landmark-based alignment implemented in TomoJ. ${ }^{27}$ The tilt series images were also background subtracted. Afterwards, they were reconstructed into a $3 \mathrm{D}$ volume using a compressed sensing algorithm based on minimization of the total variation in 3D (TVM3D) of the whole set of HAADFSTEM images. In particular, an implementation of the TVAL3D routine using the ASTRA tomography toolbox ${ }^{28}$ was employed. For visualisation and further nanometrological analysis of the reconstructed volumes, the FEI Avizo Fire Software was used. Segmentation of the reconstructed volumes was performed by a home-developed routine programmed in MATLAB as described in ref. 29.

Gas physisorption analysis was conducted at $77 \mathrm{~K}$ on the template and intermediate and final ceria products using a Micrometrics TriStar II 3020 using liquid $\mathrm{N}_{2}$. Samples were degassed at $120{ }^{\circ} \mathrm{C}$ under vacuum for at least $12 \mathrm{~h}$ prior to analysis. Micrometrics software was used to calculate Brunauer-Emmett-Teller (BET) specific surface areas (SSAs) and the Barrett, Joyner and Halenda $(\mathrm{BJH})$ pore size distribution from the adsorption branches of the isotherms. The FT-IR experiments were carried out using a Shimadzu IRAffinity-1S Fourier transform infrared spectrophotometer with highperformance LabSolutions IR software. The spectra were recorded from 4000 to $400 \mathrm{~cm}^{-1}$. A background spectrum was taken before each measurement.

\section{Results and discussion}

\subsection{Impregnation method}

Table 1 shows the SSA and pore volume of the intermediate products, SL-IP, IW-IP, DS-IP and CS-IP. In these, the ceria is expected to have formed in the pores of the OMC template but the template is expected still to be present, having not yet been removed by combustion at the higher temperature treatments of $320^{\circ} \mathrm{C} / 3 \mathrm{~h}$ and $530{ }^{\circ} \mathrm{C} / 1 \mathrm{~h}$ in air. The fact that the lowest SSA and pore volume was for CS-IP indicates the highest degree of pore filling in this sample. The other three samples showed broadly similar pore volume and SSA values to each other, which indicates a similar loading of $\mathrm{CeO}_{2}$ in these samples. The fact that the values of $D_{\mathrm{P}}$ (Table 1) are slightly smaller in the IP samples than in the parent template may be a consequence of the partial filling of some pores, with material lining the pore walls but leaving a smaller cavity along the pore axis. 
After the removal of carbon template, the resulting ceria products were subjected to physisorption, XRD and TEM analysis. Results for the IP samples, before template removal, are given in Fig. S1 and S2 (in ESI $\dagger$ ) and results for the ceria products, after template removal are presented in Fig. 1-3 and in Table 1. Fig. 1 shows the $\mathrm{N}_{2}$ adsorption isotherms and pore size distributions of SL-Ceria, IW-Ceria, DS-Ceria and CS-Ceria. The isotherm of SL-Ceria shows a typical Type IV shape with a Type $\mathrm{H} 1$ hysteresis loop at relative pressure $c a$. $0.65-0.8$ indicating the presence of regular mesopores. The uptake at relative pressure $0.8-1.0$ indicates inter-particle porosity. ${ }^{22}$ The pore size distribution of this sample confirms the presence of both uniform mesopores and inter-particle voids, respectively, by showing a relatively large peak centred at a $D_{\mathrm{P}}$ of around $8.8 \mathrm{~nm}$ followed by a broad peak at larger pore size. ${ }^{21}$ The isotherms of DS-Ceria and CS-Ceria are typical of Type IV with hysteresis loops intermediate between Type $\mathrm{H} 1$ and Type $\mathrm{H} 3$. Pore size distributions show maxima at $12.4 \mathrm{~nm}$ and $8.9 \mathrm{~nm}$, respectively, for DS-Ceria and CS-Ceria followed by broad peaks at higher pore size corresponding to inter-particle voids. In the case of IW-Ceria, the two hysteresis loops were not so obvious. The corresponding pore size distribution has a maximum at $10.4 \mathrm{~nm}$ followed by a broader feature at larger diameters. The first peak, which corresponds to the presence of uniform mesopores, is much smaller for DS-, CS- and IW-Ceria than for the SL-Ceria. These results indicate that the SL method gave rise to the best yield of mesoporous product.

It should be noted that the values of $D_{\mathrm{P}}$ (Table 1) for the ceria products are not directly comparable with those of the IP materials or the parent OMC template since, in the latter, $D_{\mathrm{P}}$ relates to cylindrical pores whereas in the ceria products the pores are the spaces between ordered cylindrical ceria nanorods and hence are not cylindrical pores themselves.

The SSAs and pore volumes of all these samples and for the carbon template are given in Table 1 . It can be seen that all mesoporous ceria samples had much lower values of SSA and pore volume than their parent carbon template. This is mainly due to the large difference in the densities of $\mathrm{CeO}_{2}\left(7.22 \mathrm{~g} \mathrm{~cm}^{-3}\right)$ and carbon $\left(2.26 \mathrm{~g} \mathrm{~cm}^{-3}\right) .{ }^{22}$ The values for the SSA and the pore volume were 107-125 $\mathrm{m}^{2} \mathrm{~g}^{-1}$ and $0.35-0.41 \mathrm{~cm}^{3} \mathrm{~g}^{-1}$,
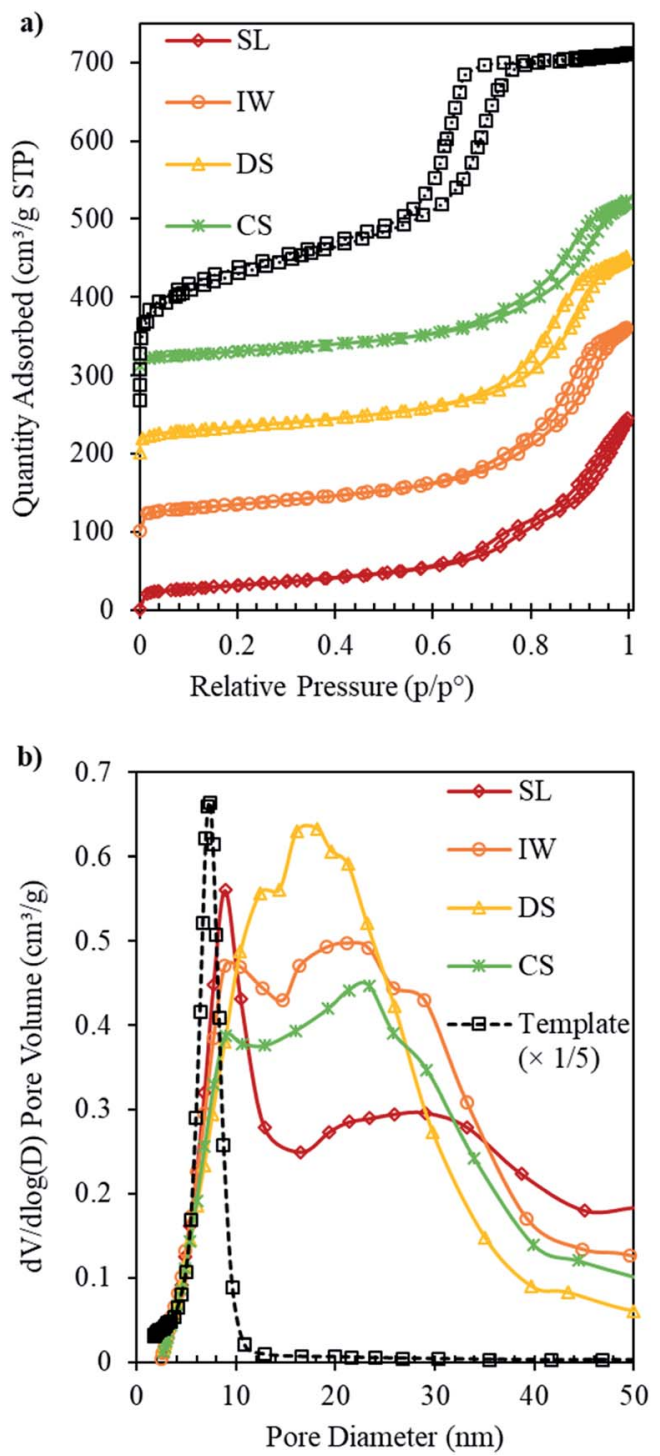

Fig. 1 Nitrogen physisorption isotherms (a) and pore size distributions (b) of SL-Ceria, IW-Ceria, CS-Ceria and DS-Ceria. The isotherms of IW-Ceria, CS-Ceria, the carbon template and DS-Ceria are off-set vertically by $100,200,250$ and $300 \mathrm{~cm}^{3} \mathrm{~g}^{-1}$, respectively. In (b) the values for the carbon template are divided by 5 for clarity.

Table 1 Textural parameters of samples made using SL, IW, DS and CS methods ${ }^{a}$

\begin{tabular}{|c|c|c|c|c|c|c|c|}
\hline Sample ID & $\operatorname{SSA}\left(\mathrm{m}^{2} \mathrm{~g}^{-1}\right)$ & $\begin{array}{l}V_{\mathrm{P}} \\
\left(\mathrm{cm}^{3} \mathrm{~g}^{-1}\right)\end{array}$ & $\begin{array}{l}V_{\text {micro }} \\
\left(\mathrm{cm}^{3} \mathrm{~g}^{-1}\right)\end{array}$ & $D_{\mathrm{P}}(\mathrm{nm})$ & $a(\mathrm{~nm})$ & $D_{\mathrm{XRD}}(\mathrm{nm})$ & $D_{\mathrm{R}}(\mathrm{nm})$ \\
\hline Template & 614 & 0.67 & 0.04 & 7.3 & 13.7 & & \\
\hline IW-IP & 316 & 0.26 & 0.04 & 6.9 & & 6.1 & \\
\hline DS-IP & 336 & 0.27 & 0.05 & 6.7 & & 6.4 & \\
\hline CS-IP & 249 & 0.20 & 0.04 & 6.5 & & 6.4 & \\
\hline DS-Ceria & 124 & 0.40 & 0.01 & 12.4 & & 8.4 & 7.5 \\
\hline CS-Ceria & 107 & 0.35 & 0.01 & 8.9 & & 8.7 & 7.9 \\
\hline
\end{tabular}

${ }^{a}$ SSA: specific surface area; $V_{\mathrm{P}}$ : pore volume; $V_{\text {micro: }}$ micropore volume; $D_{\mathrm{P}}$ : pore diameter at maxima of pore size distribution (all from gas physisorption experiments); $a$ : unit cell parameter $=\left(2 d_{100} / \sqrt{ } 3\right) ; D_{\mathrm{XRD}}$ : crystallite size calculated from peak (111) in WAXRD; $D_{\mathrm{R}}$ : rod diameter from TEM. 


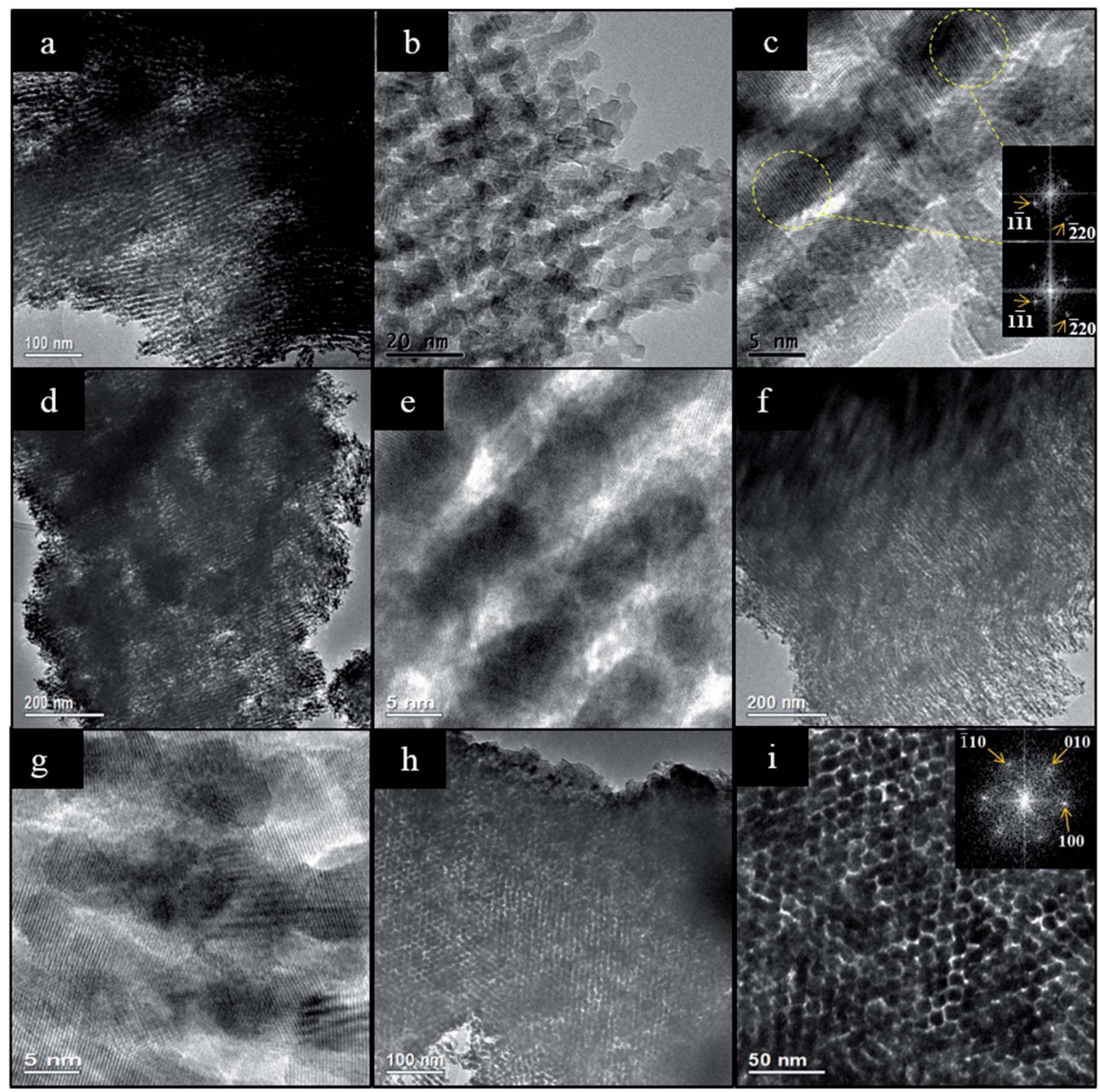

Fig. 2 Intermediate and high magnification TEM images of: (a-c) SL-Ceria; (d and e) IW-Ceria; ( $f$ and g) DS-Ceria; (all viewed along the [110] direction) and (h and i) CS-Ceria (viewed along the [001] direction of the mesopore structure) with DDP of the mesopore structure inset. The DDPs of the regions indicated in (c) can be indexed to the crystal lattice of ceria viewed along the [112] zone axis and are aligned, showing that relatively long-range order is present in the nanorods. Further high resolution images of CS-Ceria are given in Fig. 9.

respectively. The values of SSA were less than, while the values for pore volume were comparable to, the corresponding values of SSA $\left(148 \mathrm{~m}^{2} \mathrm{~g}^{-1}\right)$ and pore volume $\left(0.42 \mathrm{~cm}^{3} \mathrm{~g}^{-1}\right)$ of mesoporous ceria made using a CMK-3 template by Tiemann and coworkers. $^{22}$

The TEM analysis confirms the presence of mesostructure having long range order in the ceria samples made by all four impregnation methods. 2-D hexagonally-ordered arrays of rodlike nanostructures were found distributed throughout each sample, and this was especially marked in the case of SL-Ceria. Fig. 2 shows representative TEM images of SL-Ceria, DS-Ceria, SL-Ceria and IW-Ceria viewed along the [110] direction and of
CS-Ceria viewed along the [001] direction of the 2D hexagonal mesostructure. The images of the CS-Ceria sample and the inset Digital Diffraction Pattern (DDP) clearly show the hexagonal arrangement of ceria rods. This - and the similarity in diameter of the pores in the template and the rods in the ceria product $\left(D_{\mathrm{P}}\right.$ and $D_{\mathrm{R}}$, respectively, in Table 1 ) - confirms that the ceria materials are indeed inverse replicas of the hexagonal OMC template. The structure of the template is described extensively elsewhere. ${ }^{26}$ Although most of the particles in all four samples contained a uniform arrangement of nanorods, discontinuities in rod formation were observed occasionally in the cases of all four samples. Crystal lattice planes at spacings consistent with 
those of ceria are visible in the high magnification images shown in Fig. 2c, e and $\mathrm{g}$ and reveal the crystalline nature of these materials. DDPs were obtained from two regions of the same nanorod in Fig. 2c. These diffraction patterns can be indexed to the cubic ceria lattice viewed along the [112] zone axis and are aligned, the spots being in the same angular orientation. This shows that single crystalline regions can extend over relatively large distances within the nanorods. The average ceria rod diameters for all samples are given in Table 1 and are seen to be slightly larger - at 7.5 to $8.1 \mathrm{~nm}$ - than the pore diameter of the carbon template $(7.3 \mathrm{~nm})$ used. This indicates that the ceria nanorods grew in diameter during or after the removal of the carbon template, when they were exposed to high temperatures and oxidising conditions.

In the SAXRD patterns, the SL-Ceria shows a peak around $2 \theta$ $=0.8^{\circ}$ which could be indexed as the reflection from the (100) planes (with $d_{100} \sim 10.9 \mathrm{~nm}$ ) of the 2-D hexagonal mesoporous structure. The unit cell parameter was calculated to be $12.6 \mathrm{~nm}$ which is less than that of the carbon template $(13.7 \mathrm{~nm})$. This indicates that shrinkage of the unit cell occurred on going from template to product. The other three samples show less clear indications of a similar peak.

The WAXRD analysis showed that all four ceria samples are single phase ceria with the fluorite structure (Fm3m, ICDD 431002) (Fig. 3) with the expected unit cell dimension $\sim 5.42 \AA$ (see Table S1 in ESI $\dagger$ ). The peaks are broad and the crystallite sizes
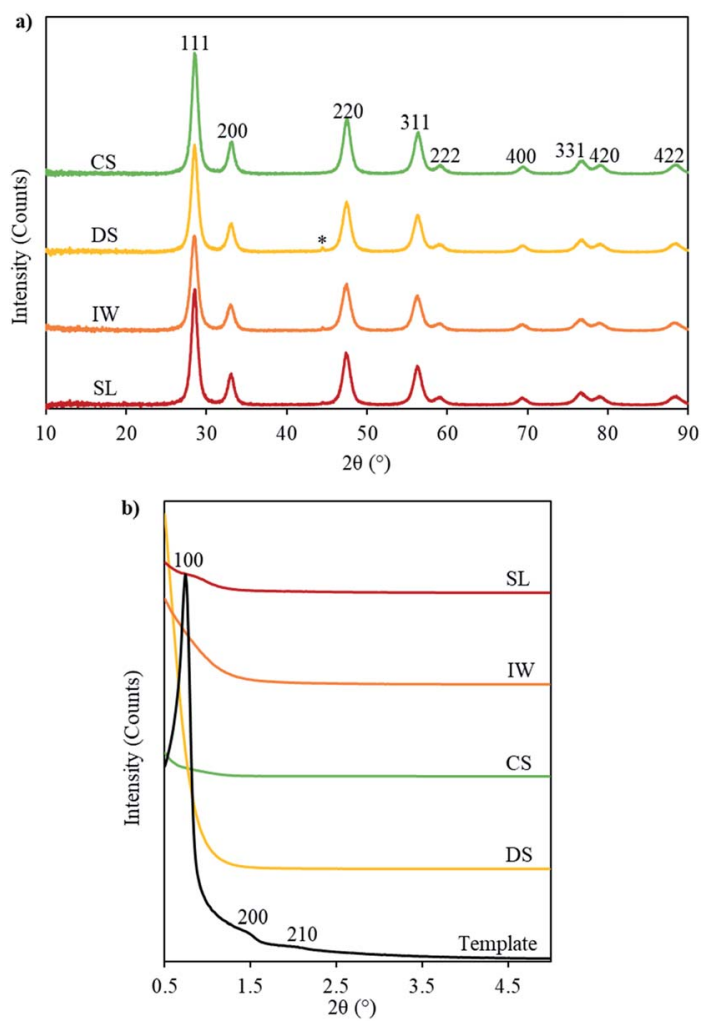

Fig. 3 WAXRD (a) and SAXRD (b) patterns of ceria products prepared using the method indicated. The small peak at about $45^{\circ}$ is due to the sample holder (main peak of $\mathrm{Fe}$ ). The plots are shifted vertically for clarity.

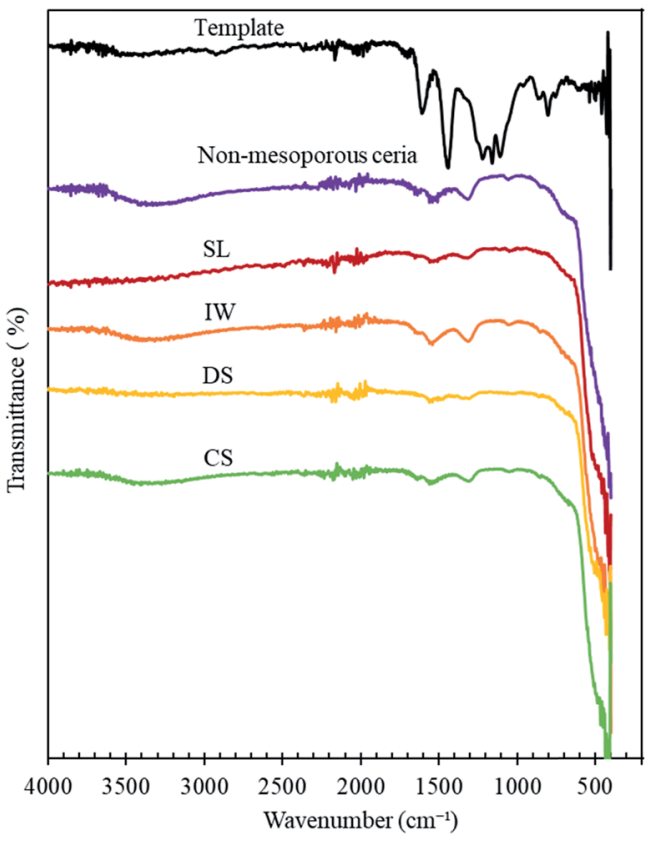

Fig. 4 FT-IR spectra of the standard carbon template, non-mesoporous ceria and mesoporous ceria products made using the methods indicated. The plots are shifted on the $y$-axis for clarity.

could be calculated using Scherrer's formula (Table 1), as they also were for the IP samples (patterns not shown). Crystallite sizes were generally smaller in the IP samples - when the carbon template was still present - than in the ceria products, in which the template had been removed. This indicates that the ceria nanocrystals underwent thermally-activated growth during or after template removal. This result corresponds well with the finding from TEM that the diameters of the ceria nanorods were generally slightly larger than the pores of the carbon template in which they nucleated and began to form.

The FT-IR patterns of the ceria products are similar to those of a non-mesoporous ceria sample (Fig. 4 and Table 2) except that water peaks between $3100-3600 \mathrm{~cm}^{-1}$ and around $1640 \mathrm{~cm}^{-1,30-34}$ are absent in SL-Ceria and DS-Ceria. The peaks around 1540 and $1050 \mathrm{~cm}^{-1}$ are in the region reported to correspond to carbonate surface species $\left(1540 \text { to } 1040 \mathrm{~cm}^{-1}\right)^{31}$ which form on ceria from the air - and the peak at around $1300 \mathrm{~cm}^{-1}$ may also be a carbonate peak or may relate to the $\mathrm{N}-\mathrm{O}$ stretch due to presence of residual nitrate from the sample preparation (reported at $1384 \mathrm{~cm}^{-1}$ ). ${ }^{31}$ The very large feature at around $550 \mathrm{~cm}^{-1}$ can be assigned to the Ce-O stretch of the

Table 2 IR peaks observed for non-mesoporous ceria and the mesoporous ceria products made using the methods indicated

\begin{tabular}{lcrrrrrr}
\hline Sample ID & \multicolumn{2}{l}{ IR peaks $\left(\mathrm{cm}^{-1}\right)$} \\
\hline Non-mesoporous & 3305 & 1638 & 1535 & 1342 & 1065 & 841 \\
SL & & & 1537 & 1320 & 1111 & 1049 & 848 \\
IW & 3360 & 1641 & 1547 & 1308 & 1047 & 848 \\
DS & & 2046 & & 1537 & 1325 & 1024 & 856 \\
CS & 3404 & & 1539 & 1305 & 1051 & 858
\end{tabular}


$\mathbf{a}$

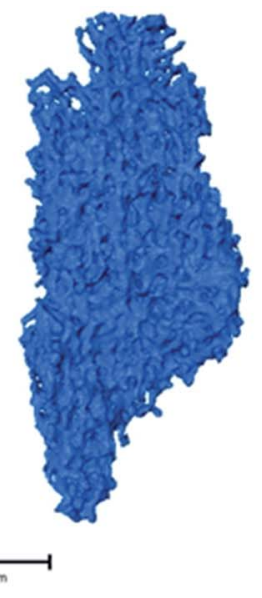

b

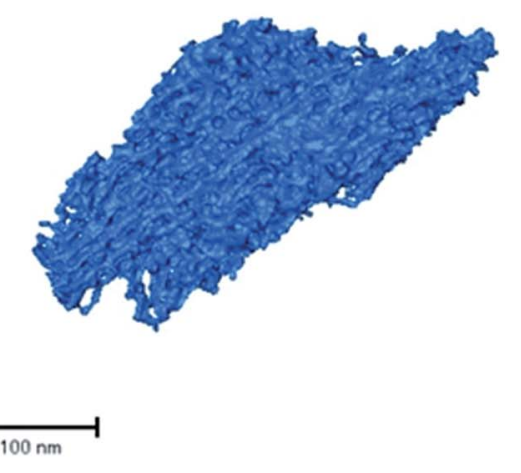

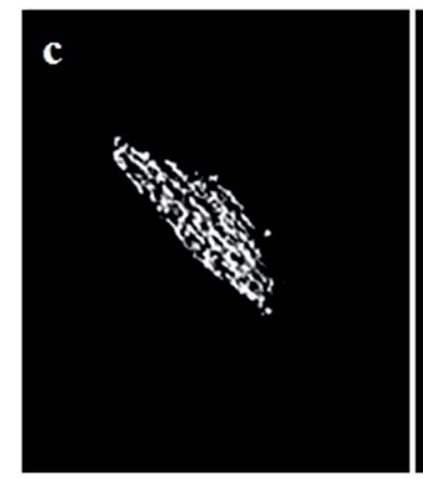
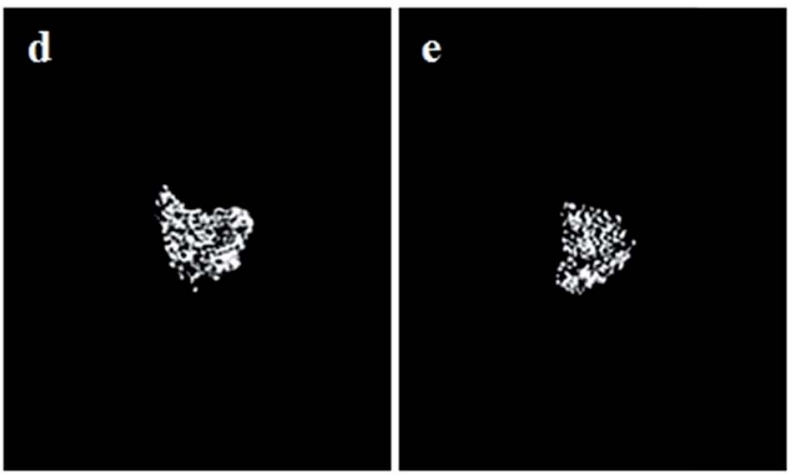

Fig. 5 Electron tomography results for the SL-Ceria sample: ( $a$ and b) perspective views of the rendered volume obtained after reconstruction; (c-e) orthoslices through the reconstructed volume in directions parallel (c) and perpendicular ( $d$ and e) to the axes of the $\mathrm{CeO}_{2}$ nanorods.

ceria lattice. ${ }^{31}$ Importantly, Fig. 4 shows that the IR spectrum of the carbon template does not match with the ceria products, indicating that the template was completely removed from the products.

Finally, a complementary 3D morphological characterisation of the solids by ET was performed. As an illustration, Fig. 5a and b show two perspective views of the rendered volume reconstructed from the tilt series for the SL-Ceria sample. The ET data show the complex 3D morphology of the mesoporous solid. As 2D TEM images suggest, the sample is not made up of isolated $\mathrm{CeO}_{2}$ rods, as might initially be expected from a template with isolated cylindrical channels, but, instead, is constituted of a network of thin rods interconnected in 3D in a complex fashion. These features are better appreciated in the orthoslices shown in Fig. 5c-e. These correspond to sections, just one voxel thick ( $\approx 0.98 \mathrm{~nm}$ ), throughout the volume of the particle shown in the rendered image. In particular, Fig. $5 \mathrm{c}$ corresponds to a section through a plane along the direction of the rods and Fig. 5d and e along their perpendicular. Both orthoslices clearly evince the mesoporous nature of the solid, while the rod-like shape of ceria particles is better observed in Fig. 5c. The width of the rods falls in the range 5-10 $\mathrm{nm}$ and the pores are roughly $7 \mathrm{~nm}$ across, in good agreement with previous TEM and physisorption results. However, another important aspect revealed by the $3 \mathrm{D}$ reconstruction is that, due to the connectivity between different rods, the pore system does not consist of simple, straight channels but of rather tortuous, nanometrescale, paths.

To check the accuracy of the ET results, the $3 \mathrm{D}$ reconstructions were segmented using an improved procedure developed by the Cádiz group. ${ }^{29}$ This process allowed us to separate the volume of the reconstruction corresponding to the solid from that of the background. Once segmented, and using the density of $\mathrm{CeO}_{2}\left(7.22 \mathrm{~g} \mathrm{~cm}^{-3}\right)$ the specific surface area of the material was estimated, and a value of roughly $100 \mathrm{~m}^{2} \mathrm{~g}^{-1}$ was obtained, which is in very good agreement with the macroscopic measurement. Therefore, the morphological details revealed by the reconstruction illustrated in Fig. 5 can be considered representative of the sample as a whole. In any case, additional reconstructions from other particles of the same sample can be found in the ESI (Fig. S3-S5†).

The nanorods themselves appear to have a somewhat rough morphology in ET, rather than being perfect, smooth cylinders. This is logical given the nature of their formation within the pores of the OMC template and the fact that the rods are crystalline with many small exposed crystal facets, as seen by TEM. The SSA of an ideal ceria mesopore structure with smooth, dense $\left(7.22 \mathrm{~g} \mathrm{~cm}^{-3}\right)$ cylindrical nanorods of diameter, $7.9 \mathrm{~nm}$, and with mesopore unit cell dimension, $12.6 \mathrm{~nm}$, (values obtained experimentally for SL-Ceria, Table 1) was calculated to be 
$70.1 \mathrm{~m}^{2} \mathrm{~g}^{-1}$. The fact that this value is lower than the experimental value $\left(111 \mathrm{~m}^{2} \mathrm{~g}^{-1}\right)$ and that obtained from the ET results $\left(\sim 100 \mathrm{~m}^{2} \mathrm{~g}^{-1}\right.$ ) would indeed be explained by the ceria nanorods having significant surface roughness. It is worth noting that this roughness would scatter X-rays non-uniformly and may explain why the peaks in the SAXRD patterns of the ceria products are not better defined.

\subsection{Effect of template calcination temperature}

One of the requirements for nanocasting metal oxides is the successful filling of pores of the mesoporous template with the precursor compound. One potential limitation of the use of OMC materials as hard templates is carbon's hydrophobic nature. After removal of the structure directing agent, the pore surfaces of OMCs possess functional groups and, because of these, have some degree of hydrophilicity. However, the presence of too many functional groups can decrease the pore size or even block the pores. Pore hydrophobicity increases as these functional groups are decomposed and removed on increasing the calcination temperature, $T_{\text {calc }}$, of the OMC materials. In order to find an appropriate value of $T_{\text {calc }}$ for a high degree of infiltration, OMCs calcined at $T_{\text {calc }}=350,400,500,600$ or $800{ }^{\circ} \mathrm{C}$ were impregnated with $\mathrm{Ce}\left(\mathrm{NO}_{3}\right)_{3} \cdot 6 \mathrm{H}_{2} \mathrm{O}$ using the $\mathrm{SL}$ method. This impregnation method was used because it is solvent free, allows ease of handling and is environmentally friendly. ${ }^{21}$

Fig. 6 presents values of SSA, pore volume and micropore volume - relative to the pristine OMC template - of the impregnated carbon templates after heating at $200{ }^{\circ} \mathrm{C}$, as a function of $T_{\text {calc }}$. Heating at $200{ }^{\circ} \mathrm{C}$ is expected to promote the crystallisation of the ceria within the pores of the OMC without

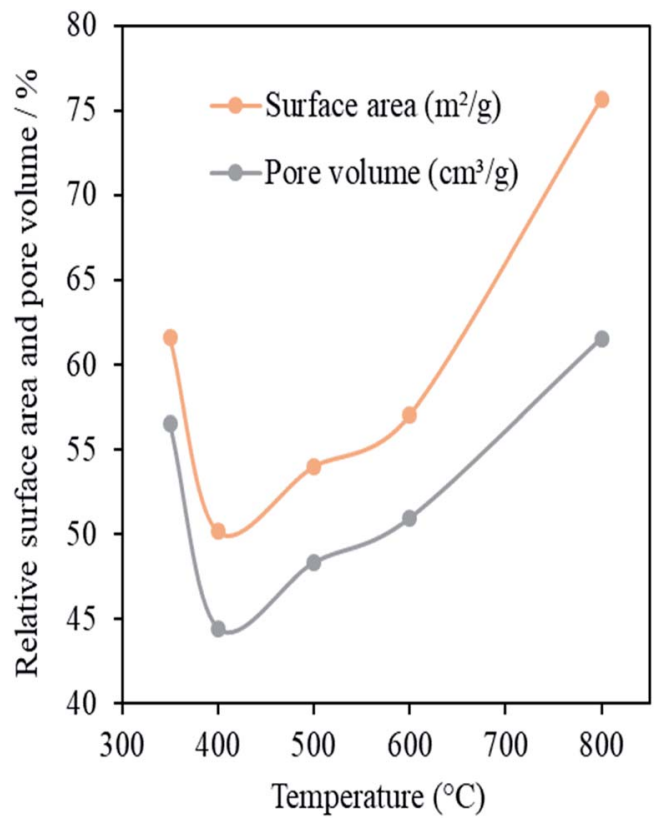

Fig. 6 Specific surface area and pore volume (relative to the SSA and pore volume of the template) vs. calcination temperature of the OMCs in ceria-impregnated samples prepared by the SL method. the template itself being removed. Therefore, these relative values give a good indication of the extent of pore filling in the impregnation step. The template calcined at $350{ }^{\circ} \mathrm{C}$ showed high relative values of SSA and pore volume after impregnation and subsequent cerium oxide formation. This indicates a low degree of pore filling. This may be because the large number of surface functional groups hindered the entry of the liquid precursor. The template calcined at $400{ }^{\circ} \mathrm{C}$ showed the highest level of pore filling as it had the lowest values of SSA and pore volume after impregnation and $\mathrm{CeO}_{2}$ formation. Further increase in $T_{\text {calc }}$ above $400{ }^{\circ} \mathrm{C}$ resulted in a clear and progressive increase in the values of these parameters. This may be attributed to increased hydrophobicity of the carbon template with increasing $T_{\text {calc }}$ which would decrease the degree of wetting of the pore walls by the liquid precursor and so decrease the efficiency of pore filling. A $T_{\text {calc }}$ of $400{ }^{\circ} \mathrm{C}$ clearly gives the best results on the basis of these data and this value was used in the preparation of the standard OMC template in this work.

The ceria products obtained by removing the carbon template were named Ceria- $T_{\text {calc }}$ where $T_{\text {calc }}$ is the temperature at which the parent template was calcined. Fig. 7a shows the WAXRD patterns of these samples which indicate their crystalline nature and show reflections typical of the fluorite-type structure of cerium oxide. Table 3 collects together textural parameters for these products and for their parent OMC
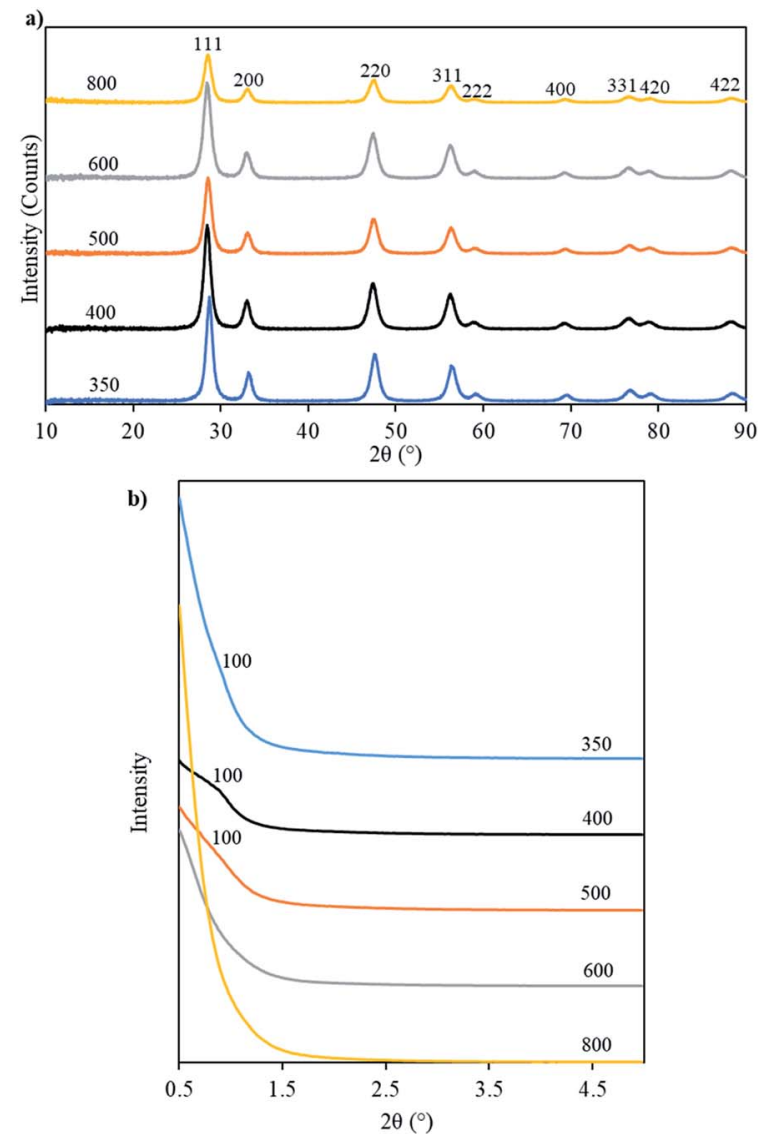

Fig. 7 WAXRD (a) and SAXRD (b) patterns of ceria prepared from OMC templates calcined at the temperatures indicated. 
Table 3 Textural parameters of the mesoporous ceria samples made using carbon templates calcined at the temperatures indicated ${ }^{a}$

\begin{tabular}{|c|c|c|c|c|c|c|c|}
\hline Sample ID & $\operatorname{SSA}\left(\mathrm{m}^{2} \mathrm{~g}^{-1}\right)$ & $V_{\mathrm{P}}\left(\mathrm{cm}^{3} \mathrm{~g}^{-1}\right)$ & $V_{\text {micro }}\left(\mathrm{cm}^{3} \mathrm{~g}^{-1}\right)$ & $D_{\mathrm{P}}(\mathrm{nm})$ & $a(\mathrm{~nm})$ & $D_{\mathrm{XRD}}(\mathrm{nm})$ & $D_{\mathrm{R}}(\mathrm{nm})$ \\
\hline Template-350 & 610 & 0.69 & 0.034 & 7.7 & 13.5 & & \\
\hline Template-400 & 608 & 0.63 & 0.053 & 7.0 & 13.0 & & \\
\hline Template-600 & 719 & 0.55 & 0.134 & 6.2 & 11.8 & & \\
\hline Template-800 & 707 & 0.52 & 0.153 & 5.7 & 11.0 & & \\
\hline Ceria-350 & 96 & 0.25 & 0.008 & 9.5 & 11.4 & 9.6 & 7.9 \\
\hline Ceria-600 & 116 & 0.31 & 0.011 & 7.6 & & 7.9 & 7.3 \\
\hline Ceria-800 & 109 & 0.26 & 0.009 & 6.8 & & 8.0 & 7.0 \\
\hline
\end{tabular}

${ }^{a}$ SSA: specific surface area; $V_{\mathrm{p}}$ : pore volume; $V_{\text {micro }}$ : micropore volume; $D_{\mathrm{P}}$ : pore diameter at maxima of pore size distribution; $a$ : unit cell parameter $=\left(2 d_{100} / \sqrt{ } 3\right) ; D_{\mathrm{XRD}}$ : crystallite size calculated from peak (111) in WAXRD; $D_{\mathrm{R}}$ : rod diameter from TEM.

templates. The crystallite sizes, calculated using Scherrer's formula, of the ceria products are seen to decrease with increasing $T_{\text {calc }}$ from 9.6 to $8.0 \mathrm{~nm}$. This indicates that - as would be expected - crystallite growth was limited by the pore diameter of the template, which also decreased as $T_{\text {calc }}$ increased (Table 3).

Fig. 7b shows the SAXRD patterns of ceria products made from templates calcined at the different values of $T_{\text {calc }}$. The patterns for Ceria-350, Ceria-400 and Ceria-500 show peaks that could be indexed as the reflection from the 100 planes of a $2 \mathrm{D}$ hexagonal mesostructure. This peak is more pronounced in the case of Ceria-400. The unit cell parameters calculated from $d_{100}$ of these three samples were smaller than the unit cell parameters of their parent template (Table 3). This shows that shrinkage of the unit cell occurred on going from the carbon templates to the mesoporous ceria products, as was described above.

Fig. 8 shows the $\mathrm{N}_{2}$ physisorption isotherms and corresponding pore size distributions for the ceria products after the removal of the template. The isotherm of Ceria-400 shows two hysteresis loops, one starting at a relative pressure of $c a$. 0.68 , which is due to capillary condensation and is typical of mesoporous materials having regular mesopores, while the
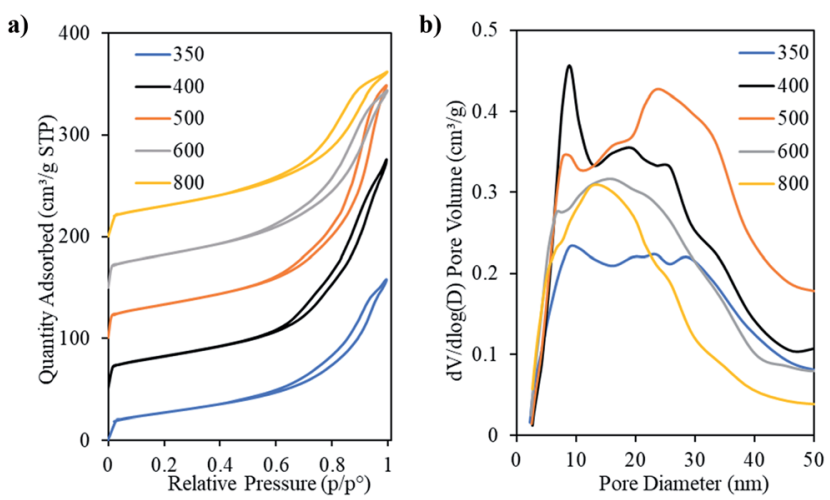

Fig. 8 Nitrogen physisorption isotherms (a) and pore size distributions (b) of ceria products made using OMC templates calcined at the temperatures indicated. The isotherms are offset vertically in steps of $50 \mathrm{~cm}^{3} \mathrm{~g}^{-1}$. other, starting at the relative pressure of $c a .0 .85$, represents the inter-particle voids (as discussed above). The presence of these two kinds of pores can be clearly seen in the corresponding pore size distribution. This shows a narrow peak centred at $c a .9 .0 \mathrm{~nm}$ and a broader feature at larger pore diameters. The two hysteresis loops are less pronounced in the cases of Ceria-350 and Ceria-500. In these samples, the peak in the pore size distribution relating to the presence of uniform mesopores is smaller than for Ceria-400. The isotherms of Ceria-600 and Ceria-800 are of Type IV with single hysteresis loops intermediate between Type H1 and Type H3. The textural parameters of these materials are given in Table 3. The Ceria350 sample had the lowest values of SSA and pore volume (96 $\mathrm{m}^{2} \mathrm{~g}^{-1}$ and $0.25 \mathrm{~cm}^{3} \mathrm{~g}^{-1}$, respectively) followed by Ceria-800 (109 $\mathrm{m}^{2} \mathrm{~g}^{-1}$ and $0.26 \mathrm{~cm}^{3} \mathrm{~g}^{-1}$, respectively). Overall, the pore volume increased on increasing the calcination temperature of the template till $T_{\text {calc }}=500{ }^{\circ} \mathrm{C}$ and then decreased at higher $T_{\text {calc. }}$. The same trend was found for the SSA and micropore volume but the difference between samples is very small in the latter. The pore diameter at the low-diameter maximum in the pore size distribution decreased smoothly on increasing template $T_{\text {calc }}$ which is logical since this also happened to the pores of the templates themselves (Table 3). Again, it should be remembered that $D_{\mathrm{P}}$ refers to cylindrical pores in the template but to the pores between cylindrical nanorods in the ceria products, and so these two sets of values cannot be directly compared.

Fig. 9 presents representative intermediate and high magnification images of the ceria products made using OMCs with varying $T_{\text {calc }}$. These confirm the presence of mesostructures with long range order. Fig. $9 \mathrm{a}-\mathrm{g}$ and $\mathrm{i}-\mathrm{k}$ show particles whose mesopore structure is viewed along the [110] direction of the hexagonal mesopore structure in the Ceria-350, Ceria-400, Ceria-500, Ceria-600 and Ceria-800 samples. The rodlike structures observed are the expected product of nanocasting from the 2D hexagonal OMC template. Fig. 9h shows a particle of Ceria-600 whose mesopore structure is viewed along the [001] direction and shows the hexagonal arrangement of the nanorods. The lattice planes of the ceria comprising the nanorods can be clearly seen in Fig. $9 \mathrm{~b}$ and $\mathrm{f}-$ as are the Moire patterns in Fig. 9d which are a consequence of the electron 


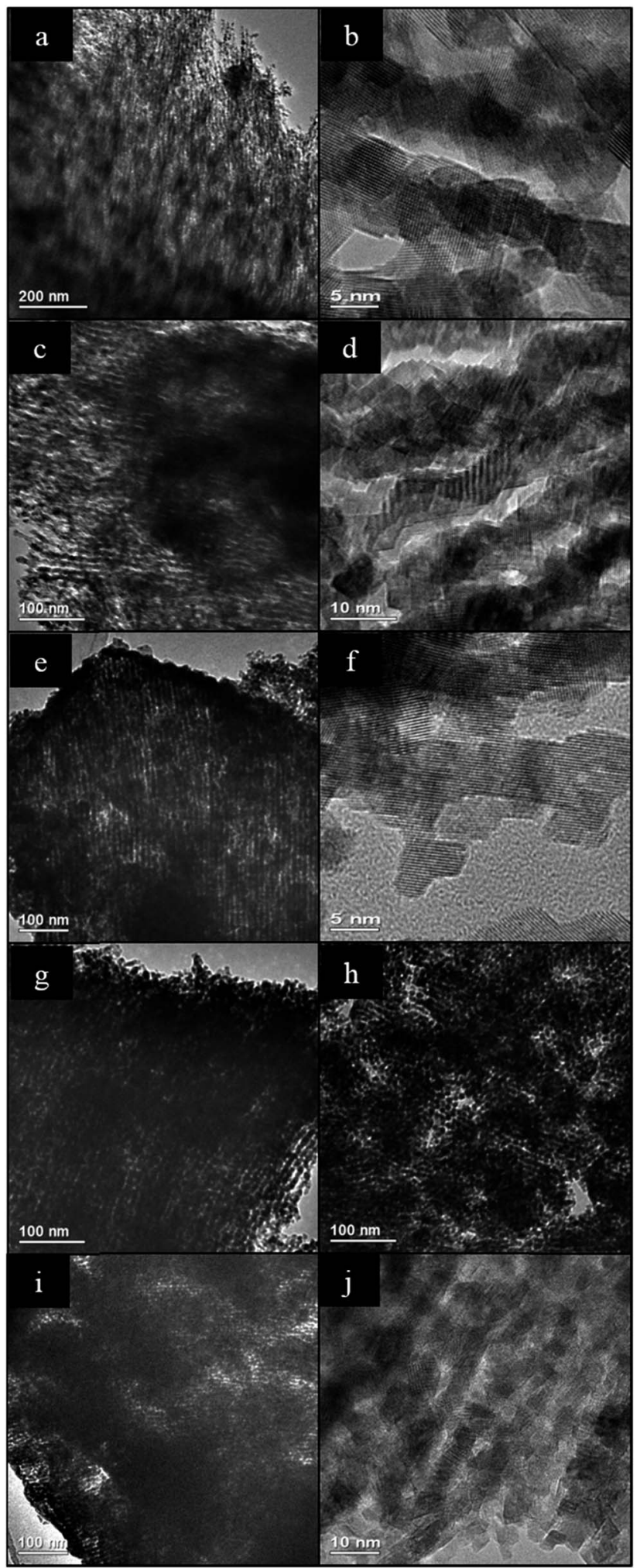

Fig. 9 TEM images at low and high magnifications of ceria mesostructures viewed along the [110] direction unless stated otherwise: (a and b) Ceria-350; (c and d) Ceria-400; (e and f) Ceria-500; (g) Ceria600; (h) Ceria-600 viewed along [001] direction; ( $\mathrm{i}$ and j) Ceria-800.

beam passing through two crystalline regions in turn. These images confirm the crystalline nature of the nanorods, and also that a particular orientation of the crystal lattice can persist over relatively large distances along the nanorods (Fig. 9b and f), as seen in Fig. 2c. To remove the carbon template, all ceria products were heated at $530{ }^{\circ} \mathrm{C}$ in air. The TEM images indicate that these materials retained their mesostructure and crystallinity which shows their thermal stability at this temperature. The average rod diameter found during TEM analysis of the ceria products was slightly larger than the pore diameter of their parent templates (Table 3) which indicates that crystal growth occurred during or after template removal (as discussed in Section 3.1). There was a decrease in average rod diameter with increasing $T_{\text {calc }}$ which is expected since the pore diameter of the carbon templates also decreased with increasing $T_{\text {calc }}$ (Table 3 ).

The physisorption analysis shows that the sample made using the carbon template sintered at $T_{\text {calc }}=400{ }^{\circ} \mathrm{C}$ gave rise to the highest loading of $\mathrm{CeO}_{2}$ of these samples after heating at $200{ }^{\circ} \mathrm{C}$ and the peak in the pore size distribution indicating the presence of regular mesopores is larger for this sample than for any other in the $T_{\text {calc }}$ series. The SAXRD pattern of this sample showed the strongest peak corresponding to the $2 \mathrm{D}$ hexagonal mesostructure and mostly ordered mesoporous material was found when the sample was examined by TEM. All these results indicate that using an OMC template prepared with $T_{\text {calc }}=$ $400{ }^{\circ} \mathrm{C}$ to prepare mesoporous ceria by nanocasting gave rise to the best results. Despite the fact that the Ceria-600 and Ceria800 were made using carbon templates that were hydrophobic in nature ${ }^{26}$ highly ordered mesoporous ceria products were still obtained, even though these had relatively small pore volumes.

\section{Conclusion}

These results show that among all impregnation methods studied, the SL method gave the narrowest pore size distribution in the small diameter mesoporous region, and the smallest amount of inter-particle voids. The sample made using this method is the only product that showed a clear peak in SAXRD corresponding to the 100 plane of the $2 \mathrm{D}$ hexagonal mesostructure. The TEM and 3D STEM analysis showed mostly ordered material in this sample. Also, this method is solvent free so allows easy handling and is environmentally friendly. So it can be concluded that this is the best of the impregnation methods studied here.

Among carbon templates calcined at different temperatures, that calcined at $400{ }^{\circ} \mathrm{C}$ gave rise to the highest loading of ceria precursor and the ceria product made using this template was found to be the best sample of the series, as shown by physisorption, SAXRD and TEM analysis.

As would be expected when considering the mechanism of nanocasting, the growth of the ceria nanocrystals and of the diameter of the ceria nanorods are both restricted by the dimensions of the pores of the OMC template. However, the rod diameters are slightly larger than the diameters of the corresponding pores in the parent OMC template, suggesting that some growth of the rods occurred during or after removal of the template. These nanorods exhibit relatively long regions in which their ceria crystal lattice remains aligned and also appear to have significant surface roughness, partly as a consequence of their crystalline nature. 
Because of their novel nanostructure and the absence of metal and halide ions - potential catalyst poisons - from their preparation route, the materials described in this paper have considerable potential for application in catalysis and electrocatalysis and these avenues are currently being investigated by the authors. Furthermore, we believe that this method could be applied generally to prepare products of a wide range of compositions.

\section{Conflicts of interest}

There are no conflicts of interest.

\section{Acknowledgements}

We thank the University of St Andrews for the PhD scholarship of FS. Electron microscopy (EM) was performed at the Electron Microscopy Facility, School of Chemistry, University of St Andrews. We thank Mr Ross Blackley for assistance with EM. STEM-HAADF ET results were obtained at the Electron Microscopy Facilities (DME SC-ICYT) of the University of Cádiz, Spain. We acknowledge funding from MINECO/FEDER (Projects MAT2017-87579-R and MAT2016-81118-P) and from the European Union's Horizon 2020 Research and Innovation Programme under grant agreement No. 823717 - ESTEEM3.

\section{References}

1 J. Roggenbuck, T. Waitz and M. Tiemann, Microporous Mesoporous Mater., 2008, 113, 575-582.

2 X. Lai, X. Li, W. Geng, J. Tu, J. Li and S. Qiu, Angew. Chem., Int. Ed., 2007, 46, 738-741.

3 Y. Ren, Z. Ma and P. G. Bruce, Chem. Soc. Rev., 2012, 41, 4909-4927.

4 M. M. Nair, H. Yen and F. Kleitz, C. R. Chim., 2014, 17, 641655.

5 D. Gu and F. Schüth, Chem. Soc. Rev., 2014, 43, 313-344.

6 B. Tian, X. Liu, H. Yang, S. Xie, C. Yu, B. Tu and D. Zhao, Adv. Mater., 2003, 15, 1370-1374.

7 Y. Wang, Y. Wang, J. Ren, Y. Mi, F. Zhang, C. Li, X. Liu, Y. Guo, Y. Guo and G. Lu, J. Solid State Chem., 2010, 183, 277-284.

8 T. Brezesinski, C. Erpen, K. I. Iimura and B. Smarsly, Chem. Mater., 2005, 17, 1683.

9 W. Yue and W. Zhou, Prog. Nat. Sci., 2008, 18, 1329-1338.

10 W. Shen, X. Dong, Y. Zhu, H. Chen and J. Shi, Microporous Mesoporous Mater., 2005, 85, 157-162.
11 S. Polarz and M. Antonietti, Chem. Commun., 2002, 25932604.

12 B. Liu and R. T. Baker, J. Mater. Chem., 2008, 18, 5200-5207.

13 Y. Ren, Z. Ma, L. Qian, S. Dai, H. He and P. G. Bruce, Catal. Lett., 2009, 131, 146-154.

14 W. Yue and W. Zhou, J. Mater. Chem., 2007, 17(47), 49474952.

15 S. C. Laha and R. Ryoo, Chem. Commun., 2003, 2138-2139.

16 A. Trovarelli, Catal. Rev. - Sci. Eng., 1996, 38, 439-520.

17 H. C. Yao and Y. F. Y. Yao, J. Catal., 1984, 86, 254-265.

18 A. Trovarelli, C. de Leitenburg, M. Boaro and G. Dolcetti, Catal. Today, 1999, 50, 353-367.

19 S. Park, J. M. Vohs and R. J. Gorte, Nature, 2000, 404, 265267.

20 P. Ji, J. Zhang, F. Chen and M. Anpoa, J. Phys. Chem. C, 2008, 112, 17809-17813.

21 W. Yue and W. Zhou, Chem. Mater., 2007, 19, 2359-2363.

22 J. Roggenbuck, H. Schafer, T. Tsoncheva, C. Minchev, J. Hanss and M. Tiemann, Microporous Mesoporous Mater., 2007, 101, 335-341.

23 H. Zhang, X. Yan and W. Li, Chin. J. Catal., 2009, 30, 10851090.

24 B. Puertolas, B. Solsona, S. Agouram, R. Murillo, A. M. Mastral, A. Aranda, S. H. Taylor and T. Garcia, Appl. Catal., B, 2010, 93, 395-405.

25 B. Solsona, R. Sanchis, A. Dejoz, T. García, L. RuizRodríguez, J. López Nieto, J. Cecilia and E. RodríguezCastellón, Catalysts, 2017, 7, 96.

26 F. Sakina and R. T. Baker, Microporous Mesoporous Mater., 2019, 289, 109622.

27 C. Messaoudi, T. Boudier, C. O. Sanchez-Sorzano and S. Marco, BMC Bioinf., 2007, 8, 288.

28 W. van Aarle, W. J. Palenstijna, J. de Beenhouwera, T. Altantzisc, S. Balsc, K. J. Batenburg and J. Sijbers, Ultramicroscopy, 2015, 157, 35.

29 M. Lopez-Haro, M. Tinoco, S. Fernández-Garcia, X. Chen, A. B. Hungria, M. Á. Cauqui and J. J. Calvino, Part. Part. Syst. Charact., 2018, 35, 1700343.

30 M. Chelliah, J. B. B. Rayappan and U. M. Krishnan, J. Appl. Sci., 2012, 12, 1734-1737.

31 M. Chelliah, J. B. B. Rayappan and U. M. Krishnan, J. Appl. Sci., 2012, 12, 1734-1737.

32 M. Palard, J. Balencie, A. Maguer and J.-F. Hochepied, Mater. Chem. Phys., 2010, 120, 79-88.

33 J. Gao, F. Gua, Y. Ma and Y. Wu, Rare Met., 2001, 20, 217-220. 34 F. I. Williams Dudley, Spectroscopic Methods in Organic Chemistry, Mc Graw-Hill Higher Education, 6th edn, 2007. 